\title{
Amplifier Placement Methods for Metropolitan WDM Ring Networks
}

\author{
An V. Tran, Member, IEEE, Rodney S. Tucker, Fellow, IEEE, and Natashia L. Boland
}

\begin{abstract}
This paper presents two new amplifier placement methods to minimize the number of amplifiers in metropolitan wavelength-division-multiplexing (WDM) rings based on integer programming techniques. The first method describes the amplifier placement problem exactly and uses a nonlinear programming solver to obtain a solution. The second method approximates some requirements in the problem and employs a linear programming solver to derive the amplifier placement solution. A new amplifier placement method for self-healing WDM rings is also reported in this paper. The new method is based on iteratively solving an amplifier placement problem for a ring network under different link failure scenarios. The solution provides a minimum number of amplifiers required to operate the self-healing ring under a normal or any single-link or single-node failure conditions.
\end{abstract}

Index Terms-Amplifier placement, integer programming, metropolitan ring, nonlinear programming, optimization, self-healing ring, wavelength-division multiplexing (WDM).

\section{INTRODUCTION}

W AVELENGTH-DIVISION-MULTIPLEXED (WDM) ring networks are important for metropolitan applications due to their bandwidth sharing and improved survivability [1]. One of the key issues associated with ring network design is the placement of optical amplifiers. In metropolitan WDM rings, a single amplifier is usually sufficient to compensate for the losses between two nodes [1]. A common approach for amplifier placement is to use one amplifier at each node [2]-[4]. This approach requires many amplifiers to operate the network. Amplifiers are expensive devices and may significantly affect the overall system cost. A high number of amplifiers also mean an increased probability of ring failure, which can potentially cause a significant interruption to service. It is therefore desirable to reduce the number of amplifiers in WDM ring networks.

Most of the studies on amplifier placement schemes to minimize the number of amplifiers [5]-[10] are concerned only with star and switched networks and do not consider a full set of constraints. Ring networks are different to star and switched networks due to the problems of ring lasing and recirculating amplified spontaneous emission (ASE) noise, which leads to amplifier saturation. Moreover, the effects of amplifier noise and

Manuscript received December 16, 2003; revised June 21, 2004.

A. V. Tran and R. S. Tucker are with the ARC Special Research Centre for Ultra-Broadband Information Networks and Australian Photonics Cooperative Research Centre, Photonics Research Laboratory, Department of Electrical and Electronic Engineering, The University of Melbourne, VIC 3010, Australia (e-mail: a.tran@ee.mu.oz.au; r.tucker@ee.mu.oz.au).

N. L. Boland is with the Department of Mathematics and Statistics, The University of Melbourne, VIC 3010, Australia (e-mail: N.Boland@ms.unimelb.edu.au).

Digital Object Identifier 10.1109/JLT.2004.836744 optical add-drop multiplexer (OADM) crosstalk on the bit-error rate (BER) of the received signals have not been considered. The self-healing WDM ring architecture also presents a problem for amplifier placement since the network has a different configuration under different failure conditions. The placement of amplifiers in the self-healing ring needs to ensure that the network is operational under normal, any link, or node failure conditions.

This paper presents two amplifier placement methods for minimizing the number of amplifiers in metropolitan single-fiber WDM rings. The first method uses nonlinear and linear constraints to describe the amplifier placement problem and is solved using a mixed-integer nonlinear programming (MINLP) solver. The second method contains only linear constraints and is solved using a mixed-integer linear programming (MILP) solver. An iterative method to optimally place amplifiers in self-healing WDM rings is also presented. The iterative method, based on the first amplifier placement method for single-fiber rings, provides a minimum number of amplifiers required to operate the self-healing ring under normal, any single-link, or single-node failure conditions.

The paper is organized as follows. The single-fiber ring-network architecture and amplifier model are described in Section II. Section III presents the amplifier placement Method 1 for single-fiber WDM rings and numerical solutions. Section IV describes the amplifier placement Method 2 together with numerical solutions. The iterative method for amplifier placement in a self-healing ring is explained in Section V. Section VI concludes the paper.

\section{NETWORK STRUCTURE}

The metropolitan WDM ring network studied here includes a simple ring without protection and a self-healing ring [4]. We begin with the simple ring shown in Fig. 1. The ring has $N$ nodes and $M$ amplifiers where $M \leq N$. In this logical-meshed ring, each pair of nodes is assumed to have one unique wavelength to communicate with each other. Hence, the number of wavelengths required for a full mesh connection is $W=N(N-$ 1)/2. Each node in the WDM ring network shown in Fig. 1 is an OADM with a multiplexer/demultiplexer (MUX/DEMUX) configuration [11] that can add/drop $(N-1)$ different wavelengths to or from all other nodes.

The optical amplifiers generally operate in the saturation region, and an accurate solution to the amplifier placement problem requires a model of this saturation. We use the following simplified amplifier gain model [12], which provides 


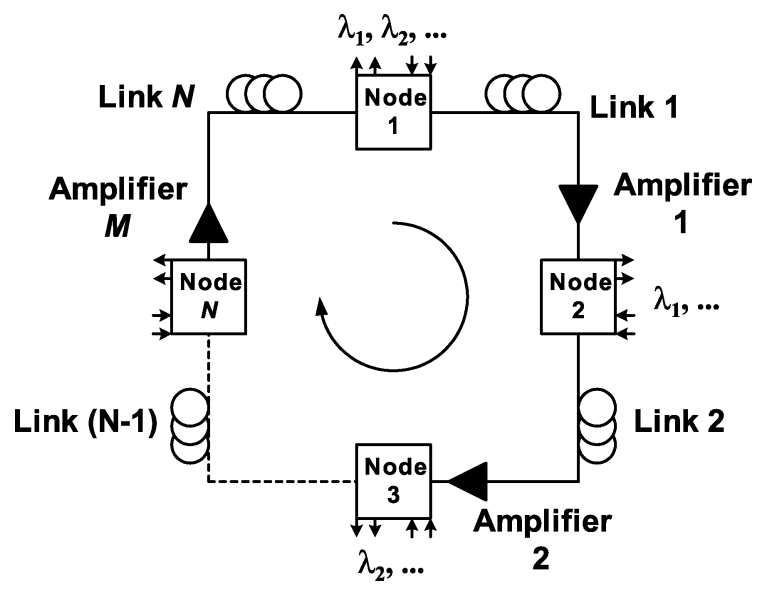

Fig. 1. Logical-meshed unidirectional WDM ring.

reasonable approximation of the amplifiers in use and enables fast computation:

$$
G=1+\frac{P_{\text {sat }}}{P_{\text {in }}} \ln \left(\frac{G_{0}}{G}\right)
$$

where $G$ is the saturated gain in linear scale, $P_{\text {sat }}$ is the internal saturation power in watts, $P_{\text {in }}$ is the total input power (across all wavelengths) in watts, and $G_{0}$ is the small-signal (unsaturated) gain in linear scale.

Gain $G$ in decibels versus total input power $P_{\text {in }}$ in decibels referred to 1 milliwatt $(\mathrm{dBm})$ is plotted as dotted lines in Fig. 2 for $P_{\text {sat }}=10 \mathrm{dBm}$ and $G_{0}=30 \mathrm{~dB}$. The gain curve is also piecewise linearly approximated and is shown by solid lines in Fig. 2. The gain value $G$ shown in Fig. 2 is the amplifier gain at the optimized pump power and erbium-doped fiber length. It is assumed in this study that the actual gain of each amplifier $\left(G^{\prime}\right)$ is upper-bounded by the gain value $G$. The actual gain $G^{\prime}$ is a design variable and is given from the amplifier placement solution.

All amplifiers are assumed to have a flat gain over the wavelengths being amplified. It is also assumed that gain saturation is a function of the total of all input powers at all wavelengths, and this is independent of wavelengths.

\section{AMPLIFIER PlacEMENT IN Single-FiBER WDM RingS: MiXed-INTEGER NONLINEAR PROGRAMMING METHOD} (METHOD 1)

The amplifier placement Method 1 involves formulating the amplifier placement problem as an MINLP problem and solving that problem. Depending on the location of the amplifier on the link, Method 1 is referred to as either Method 1A or Method 1B. In Method 1A, amplifiers are allowed to be placed anywhere on the links, and in Method 1B, amplifiers are only allowed to be placed at the end of the links.

\section{A. Problem Formulation}

The amplifier placement problem contains exact constraints to describe the network and device requirements, including requirements on transmitted and received powers, the receiver's dynamic range, the amplifier gain, the nonlinearity power limitation, the in-band crosstalk between add and drop channels at

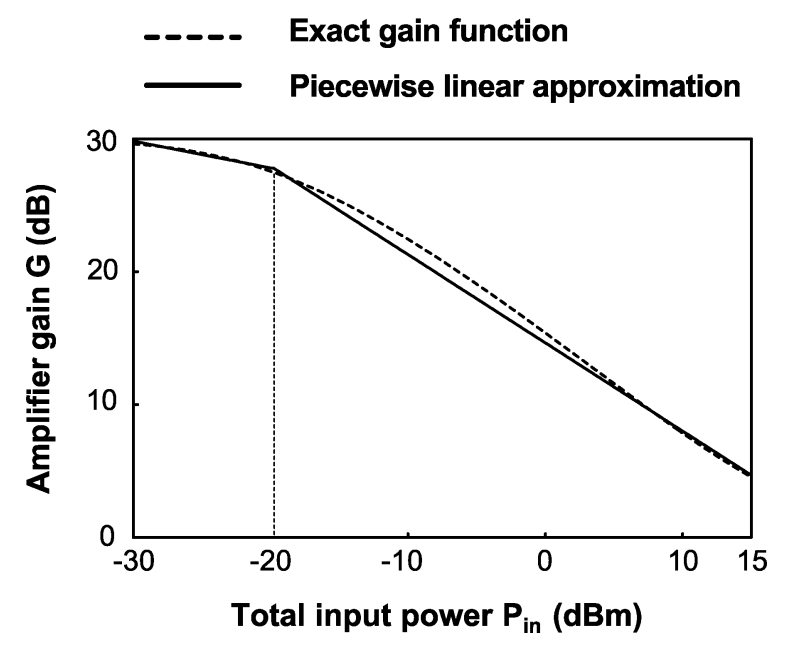

Fig. 2. Gain $G$ (in decibels) versus total input power $P_{\text {in }}$ (in $\mathrm{dBm}$ ) for $P_{\text {sat }}=$ $10 \mathrm{dBm}$ and $G_{0}=30 \mathrm{~dB}$.

TABLE I

DEVICE PARAMETERS

\begin{tabular}{c|l|c}
\hline Symbol & \multicolumn{1}{|c}{ Description } & Value \\
\hline$P_{\text {minr }}$ & $\begin{array}{l}\text { Minimum detectable signal power at a receiver (receiver } \\
\text { sensitivity) }\end{array}$ & $-30 \mathrm{dBm}$ \\
\hline$D R$ & Dynamic range of the receiver for reliable operation & $25 \mathrm{~dB}$ \\
\hline$P_{\operatorname{maxt}}$ & Maximum output power of a transmitter & $0 \mathrm{dBm}$ \\
\hline$\beta 1_{j}$ & Insertion loss for through channels at node $j$ & $10 \mathrm{~dB}$ \\
\hline$\beta 2_{j}$ & Insertion loss for drop channels at node $j$ & $5 \mathrm{~dB}$ \\
\hline$\beta 3_{j}$ & Insertion loss for add channels at node $j$ & $5 \mathrm{~dB}$ \\
\hline$I X 1_{j}$ & $\begin{array}{l}\text { Leakage at the add/drop wavelength from the input to } \\
\text { output ports at node } j\end{array}$ & $-40 \mathrm{~dB}$ \\
\hline$L X 2_{j}$ & $\begin{array}{l}\text { Leakage at the add/drop wavelength from the add to drop } \\
\text { ports at node } j\end{array}$ & $-100 \mathrm{~dB}$ \\
\hline
\end{tabular}

a node, and the received optical signal-to-noise ratio (OSNR). The objective function is to minimize the number of amplifiers in the ring network. Parameters, variables, constraints, and the objective function used in Method $1 \mathrm{~A}$ are described in detail in this section. The problem formulation used in Method 1B is very similar to that in Method 1A with the differences explicitly stated.

Unless otherwise stated, all calculations in this section are performed using a decibel scale to minimize the number of nonlinear constraints and expressions.

Parameters: The device parameters used in the problem formulation are summarized in Table I. The network parameters are summarized in Table II. $A$ (in $\mathrm{dBm}$ ) is defined as a constant to calculate the ASE noise power using 0.1-nm bandwidth and is given as follows:

$$
A=10 \log _{10}\left(2 N_{\mathrm{sp}} h \nu B_{0} 10^{3}\right)
$$

where $N_{\mathrm{sp}}=2$ is the spontaneous emission factor, $h=6.63 \times$ $10^{-34} \mathrm{~J} \times \mathrm{s}$ is the Planck's constant, $\nu=\left(3.10^{8} / 1550.10^{-9}\right) \mathrm{Hz}$ is the signal frequency, $B_{0}=12.5 \times 10^{9} \mathrm{~Hz}$ is the noise bandwidth (equivalent to $0.1 \mathrm{~nm}$ ), and $10^{3}$ is used to convert from watts to milliwatts. $B$ (in $\mathrm{dBm}$ ) is defined as a constant to calculate the ASE noise power using the system bandwidth of 20 nm determined by the MUX/DEMUX-based OADMs [13], as follows:

$$
B=10 \log _{10}\left(2 N_{\mathrm{sp}} h \nu B_{1} 10^{3}\right)
$$


TABLE II

NETWORK PARAMETERS

\begin{tabular}{|c|c|c|}
\hline Symbol & Description & Value/Unit \\
\hline$P_{\text {nonlinear }}$ & $\begin{array}{l}\text { Maximum total power allowed at any point in fiber to } \\
\text { prevent any significant nonlinear effects such as four- } \\
\text { wave mixing, self-phase modulation etc. from } \\
\text { occurring in transmission }\end{array}$ & $15 \mathrm{dBm}$ \\
\hline$A$ & $\begin{array}{l}\text { Constant to calculate ASE noise power using 0.1-nm } \\
\text { bandwidth }\end{array}$ & $\mathrm{dBm}$ \\
\hline$B$ & $\begin{array}{l}\text { Constant to calculate ASE noise power using } 20-\mathrm{nm} \\
\text { bandwidth }\end{array}$ & $\mathrm{dBm}$ \\
\hline Desired_OSNR & $\begin{array}{l}\text { Minimum OSNR allowed for good quality of received } \\
\text { signal (OSNR is defined as the ratio of the signal power } \\
\text { over the noise power in a } 0.1-\mathrm{nm} \text { bandwidth) }\end{array}$ & $20 \mathrm{~dB}$ \\
\hline MARGIN & Margin to prevent lasing in the ring & $10 \mathrm{~dB}$ \\
\hline$\alpha$ & Signal attenuation in fiber & $0.2 \mathrm{~dB} / \mathrm{km}$ \\
\hline$L_{i}$ & Length of link $i$ & $\mathrm{~km}$ \\
\hline$N$ & Number of nodes in the ring & \\
\hline$L$ & Number of links in the ring, $L=N$ & \\
\hline$W$ & $\begin{array}{l}\text { Total number of wavelengths in the ring, } \\
W=N(N-1) / 2\end{array}$ & \\
\hline$W_{i}$ & $\begin{array}{l}\text { Set of wavelengths carried on link } i\left(\lambda_{k} \in W_{i}, k=\right. \\
1, \ldots, W)\end{array}$ & \\
\hline$\left|W_{i}\right|$ & Total number of wavelengths on link $i,\left|W_{i}\right|=W$ & \\
\hline$D_{R O P}$ & Set of wavelengths dropped at node $j$ & \\
\hline$A D D_{j}$ & $\begin{array}{l}\text { Set of wavelengths added at node } j, D R O P_{j}=A D D_{j} \text { for } \\
\text { the ring shown in Fig. } 1\end{array}$ & \\
\hline$N_{O N D R O P}$ & Set of wavelengths passing through node $j$ & \\
\hline LINKS & Set of links in the ring & \\
\hline NODES & Set of nodes in the ring & \\
\hline$S C R_{i}$ & Source of link $i$, also a link, $S C R_{i} \in L I N K S$ & \\
\hline$D E S T_{i}$ & Destination of link $i$, also a link, $D E S T_{i} \in$ LINKS & \\
\hline$N O D E \_S R C_{i}$ & $\begin{array}{l}\text { Node at the beginning of link } i \text {, } \\
N O D E S R C_{i} \in N O D E S\end{array}$ & \\
\hline$\overline{N O D E \_D E S T_{i}}$ & Node at the end of link $i, N O D E D E S T_{i} \in N O D E S$ & \\
\hline
\end{tabular}

TABLE III

VARIABLES

\begin{tabular}{|c|c|c|}
\hline Symbol & Description & Unit \\
\hline$n_{i}$ & $\begin{array}{l}\text { Number of amplifiers on link } i \text {. This is a binary } \\
\text { variable. If } n_{i}=0 \text { then no amplifier is placed on link } \\
i \text {, if } n_{i}=1 \text { then there is one amplifier placed on link } i \text {. }\end{array}$ & \\
\hline$K_{i}$ & $\begin{array}{l}\text { Location of the amplifier on link } i \text { from node } \\
N O D E \_S R C_{i}\left(0 \leq K_{i} \leq L_{i}\right)\end{array}$ & $\mathrm{km}$ \\
\hline$G_{i}$ & Gain of the amplifier on link $i$ & $\mathrm{~dB}$ \\
\hline$P_{i}^{\text {tot }}$ & Total power at the input to the amplifier on link $i$ & $\mathrm{dBm}$ \\
\hline$P_{i}^{\text {tot_ase }}$ & $\begin{array}{l}\text { Total ASE noise power at the end of link } i \text { calculated } \\
\text { using a } 0.1-\mathrm{nm} \text { bandwidth. This noise power is } \\
\text { contributed from all amplifiers in the ring. }\end{array}$ & $\mathrm{dBm}$ \\
\hline$P_{i, k}$ & $\begin{array}{l}\text { Power of the channel at wavelength } \lambda_{k} \text { at the } \\
\text { beginning of link } i, \lambda_{k} \in W_{i}\end{array}$ & $\mathrm{dBm}$ \\
\hline$P_{j, k}^{x m i t}$ & $\begin{array}{l}\text { Transmitted power of the add channel at wavelength } \\
\lambda_{k} \text { at the add port of node } j, \lambda_{k} \in A D D_{j}\end{array}$ & $\mathrm{dBm}$ \\
\hline$U_{i}^{0}, U_{i}^{1}, U_{i}^{2}$ & $\begin{array}{l}\text { Non-negative variables for link } i \text { to calculate gain } G_{i} \\
\text { and will be explained later }\end{array}$ & \\
\hline$M_{i}^{0}, M_{i}^{1}$ & $\begin{array}{l}\text { Binary variables for link } i \text { to calculate gain } G_{i} \text { and } \\
\text { will be explained later }\end{array}$ & \\
\hline
\end{tabular}

where $B_{1}=2.5 \times 10^{12} \mathrm{~Hz}$ is the system bandwidth (equivalent to $20 \mathrm{~nm}$ ).

Variables: The variables are summarized in Table III. In Method 1B, since amplifiers are placed at the end of the links, $K_{i}$ is not a variable and is equal to $L_{i}$. Some of the variables shown in Table III are illustrated in Fig. 3. Link $i$ is from node $\mathrm{NODE}_{-} \mathrm{SRC}_{i}$ to node NODE_DEST ${ }_{i} . \lambda_{k}$ is the wavelength being added at node NODE_SRC $i, \lambda_{k} \in \mathrm{ADD}_{\mathrm{NODE}_{-} \mathrm{SRC}_{i}} \cdot \lambda_{m}$ is the wavelength present on link $i, \lambda_{m} \in W_{i}$.

Constraints:

a) Constraints for total power: The total power in watts is calculated as the sum of all channel powers and the total ASE noise power on that link. The total ASE noise power at the beginning of link $i$ is equal to $P_{\mathrm{SRC}_{i}}^{\mathrm{tot}}-\beta 1_{\mathrm{NODE} \_\mathrm{SRC}_{i}}-A+B$ where

$P_{\mathrm{SRC}_{i}}^{\mathrm{tot}}$ ise the total ASE noise power at the end of the previous link $\mathrm{SRC}_{i}$;

$\beta 1_{\mathrm{NODE}_{-} \mathrm{SRC}_{i}}$ is the insertion loss for through channels at node NODE_SRC ${ }_{i}$.

The total power at the input to the amplifier in $\mathrm{dBm}\left(P_{i}^{\text {tot }}\right)$ is

$$
\begin{aligned}
& P_{i}^{\mathrm{tot}}=10 \log _{10}\left[\left(\sum_{k=1}^{W} 10^{\frac{P_{i, k}}{10}}\right)\right. \\
& \left.+10 \frac{P_{\mathrm{SRC}_{i}}^{\mathrm{tot}_{i} \mathrm{ase}_{i}-\beta 1_{\mathrm{NODE}} \mathrm{SRC}_{i}-A+B}}{10}\right]-\alpha K_{i} .
\end{aligned}
$$

For Method 1B, where amplifiers are placed at the end of the links, $K_{i}=L_{i}$, Constraint (4) becomes

$$
\begin{aligned}
P_{i}^{\mathrm{tot}}=10 \log _{10} & {\left[\left(\sum_{k=1}^{W} 10^{\frac{P_{i, k}}{10}}\right)\right.} \\
+ & 10^{\left.\frac{P_{\mathrm{SRC}_{i}}^{\mathrm{tot}_{\text {ase }}}-\beta 1_{\mathrm{NODE} \_\mathrm{SRC}_{i}}-A+B}{10}\right]-\alpha L_{i} .}
\end{aligned}
$$




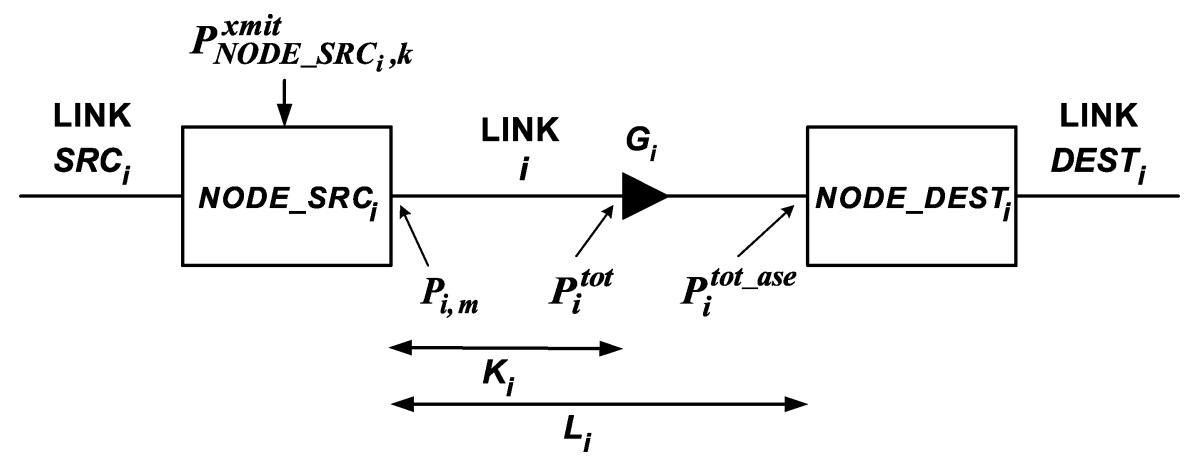

Fig. 3. Variables on link $i$.

Both Constraint (4) in Method 1A and Constraint (4b) in Method $1 \mathrm{~B}$ are highly nonlinear constraints. The linear approximation of Constraints (4) and (4b) is studied in Section IV, which leads to the formulation of the MILP problem used in the amplifier placement Method 2.

The total power at any point on link $i$ needs to be lower than a maximum value $P_{\text {nonlinear }}$ to prevent any significant nonlinear effects such as four-wave mixing, self-phase modulation, etc., from occuring. Hence, both the total power at the beginning of the link $\left(P_{i}^{\text {tot }}+\alpha K_{i}\right)$ and the total power after the amplifier $\left(P_{i}^{\text {tot }}+G_{i}\right)$ need to be less than or equal to $P_{\text {nonlinear }}$

$$
\begin{aligned}
P_{i}^{\text {tot }}+\alpha K_{i} & \leq P_{\text {nonlinear }} \\
P_{i}^{\text {tot }}+G_{i} & \leq P_{\text {nonlinear }} .
\end{aligned}
$$

For Method 1B, Constraint (5) becomes:

$$
P_{i}^{\text {tot }}+\alpha L_{i} \leq P_{\text {nonlinear }}
$$

b) Constraints for amplifier gain: The piecewise linear approximation of amplifier gain $G$ as a function of total input power $P_{\text {in }}$ is shown by the solid lines in Fig. 2. The logic to calculate gain $G_{i}$ of the amplifier on link $i$ as a function of total input power $P_{i}^{\text {tot }}$ is derived from the piecewise linear approximation as follows:

$$
\begin{aligned}
\text { If }\left(P_{i}^{\text {tot }} \leq-20\right), & \text { then } \quad G_{i} \leq-0.2 P_{i}^{\text {tot }}+23.7 \\
\text { else if }\left(P_{i}^{\text {tot }} \leq 15\right), & \text { then } \quad G_{i} \leq-0.66 P_{i}^{\text {tot }}+14.5 \\
\text { else, }, & \text { then } \quad G_{i}=0 .
\end{aligned}
$$

The above logic is modeled as a mixed-integer program using standard techniques for modeling piecewise linear function [14], modified to take into account the fact that there may or may not be an amplifier on the link $\left(n_{i}=1\right.$ or $\left.n_{i}=0\right)$. This enables $G_{i}$ to be approximated as a piecewise linear function of $P_{i}^{\text {tot }}$. Constraints for $G_{i}$ are

$$
\begin{aligned}
& G_{i} \leq 29.7 U_{i}^{0}+27.7 U_{i}^{1}+4.6 U_{i}^{2} \\
& U_{i}^{0}+U_{i}^{1}+U_{i}^{2}=n_{i} \\
& (-30) U_{i}^{0}+(-20) U_{i}^{1}+15 U_{i}^{2} \geq P_{i}^{\text {tot }}-P_{\text {nonlinear }}\left(1-n_{i}\right) \\
& (-30) U_{i}^{0}+(-20) U_{i}^{1}+15 U_{i}^{2} \leq P_{i}^{\text {tot }}-P_{\operatorname{minr}}\left(1-n_{i}\right) \\
& U_{i}^{0} \leq M_{i}^{0} \\
& U_{i}^{2} \leq M_{i}^{1} \\
& M_{i}^{0}+M_{i}^{1}=1
\end{aligned}
$$

where $U_{i}^{0}, U_{i}^{1}$, and $U_{i}^{2}$ are nonnegative variables for link $i$ to evaluate which section of the piecewise linear approximation $G_{i}$ is in; and $M_{i}^{0}$ and $M_{i}^{1}$ are binary variables for link $i$, being either 0 or 1, to ensure that $G_{i}$ is in one piecewise linear section only.

Constraint (7) indicates that $G_{i}$ is upper-bounded by a piecewise linear function of $P_{i}^{\text {tot }}$, which consists of two linear sections. These sections correspond to the break points shown in Fig. 2. The values of 29.7, 27.7, and 4.6 shown in Constraint (7) correspond to the gain value at the input power of -30 , -20 , and $15 \mathrm{dBm}$, respectively, shown in Fig. 2. Constraint (8) ensures that if there is no amplifier on link $i, U_{i}^{0}, U_{i}^{1}$, and $U_{i}^{2}$ are all equal to 0 , and consequently, gain $G_{i}$ is equal to 0 . Otherwise, the sum of $U_{i}^{0}, U_{i}^{1}$, and $U_{i}^{2}$ is equal to 1 , and gain $G_{i}$ is in one of the linear sections. Constraints (9) and (10) ensure that if $n_{i}=1$, then the total input power $P_{i}^{\text {tot }}=$ $(-30) U_{i}^{0}+(-20) U_{i}^{1}+15 U_{i}^{2}$ and $G_{i}$ is calculated using Constraints (7)-(9). Otherwise, if $n_{i}=0$, then $P_{i}^{\text {tot }}$ is restricted to be between $P_{\text {minr }}$ and $P_{\text {nonlinear }}$. Constraints (11)-(13) ensure that gain $G_{i}$ can only be in one piecewise linear section.

c) Constraint for total ASE noise: In terms of a linear (in watts, rather than decibels) power scale, the total ASE noise power at the end of link $i$ is the sum of the ASE noise power from the amplifier on link $i$ and the total ASE noise power from the previous link. The total ASE noise power, and hence the noise figure of the amplifier, is a function of the amplifier gain and input power. $P_{i}^{\text {tot_ase }}$ is given by

$$
\begin{aligned}
P_{i}^{\text {tot_ase }}=10 \log _{10} & {\left[\frac{10^{\frac{A}{10}\left(10^{\frac{G_{i}}{10}}-1\right)}}{10^{\left(\frac{\alpha\left(L_{i}-K_{i}\right)}{10}\right)}}\right.} \\
& \left.+10^{\frac{P_{\mathrm{SRC}_{i}}^{\text {tot ase }_{2}}+G_{i}-\beta 1_{\mathrm{NODE}_{\text {SRC }}-\alpha L_{i}}}{10}}\right] .
\end{aligned}
$$

For Method 1B where $K_{i}=L_{i}$, Constraint (14) becomes

$$
\begin{aligned}
P_{i}^{\text {tot_ase }}=10 \log _{10} & {\left[10^{\frac{A}{10}}\left(10^{\frac{G_{i}}{10}}-1\right)\right.} \\
+ & 10^{\left.\frac{P_{\mathrm{SRC}_{i}}^{\mathrm{tot}_{2}}+G_{i}-\beta 1_{\mathrm{NODE}_{\text {SRC }}-\alpha L_{i}}}{10}\right] .}
\end{aligned}
$$

d) Constraint for powers between adjacent links: If the channel at wavelength $\lambda_{k}$ is not dropped at node NODE_DEST $i$, the channel power at the beginning of the next link $\left(P_{\mathrm{DEST}_{i}, k}\right)$ 
is related to the power at the beginning of the current link $i\left(P_{i, k}\right)$ as follows:

$$
\begin{aligned}
& P_{\mathrm{DEST}_{i}, k}=P_{i, k}+G_{i}-\alpha L_{i}-\beta 1_{\mathrm{NODE}_{\mathrm{DEST}}} \\
& \forall \lambda_{k} \in \text { NONDROP }_{\text {NODE_DEST }} \text {. }
\end{aligned}
$$

e) Constraint for OSNR: If the channel at wavelength $\lambda_{k}$ is dropped at node NODE_DEST $i$, the received OSNR of this signal needs to be above a minimum value (Desired_OSNR) to achieve a desired BER. The constraint for OSNR is

$$
\begin{aligned}
P_{i, k}+G_{i}-\alpha L_{i}-P_{i}^{\text {tot_ase }} & \geq \text { Desired_OSNR } \\
& \forall \lambda_{k} \in \text { DROP }_{\text {NODE_DEST }_{i} .}
\end{aligned}
$$

f) Constraints for transmitted power: If the channel at wavelength $\lambda_{k}$ is added at node NODE_DEST $i$, the channel power at the beginning of the next link $\left(P_{\mathrm{DEST}_{i}, k}\right)$ is related to the transmitted power from the node

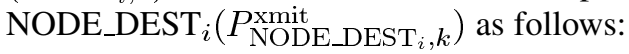

$$
\begin{aligned}
P_{\mathrm{DEST}_{i}, k}=P_{\mathrm{NODE}_{\mathrm{NDEST}}, k}^{\mathrm{xmit}}-\beta 3_{\mathrm{NODE}_{2} \mathrm{DEST}_{i}} \\
\forall \lambda_{k} \in \mathrm{ADD}_{\mathrm{NODE}_{\_D E S T}} .
\end{aligned}
$$

The transmitted power is limited by a maximum value as follows:

$$
P_{\mathrm{NODDE}_{\mathrm{DDEST}}, k}^{\mathrm{xmit}} \leq P_{\text {maxt }} \quad \forall \lambda_{k} \in \mathrm{ADD}_{\mathrm{NODE}_{\mathrm{NDEST}}} .
$$

g) Constraints for received power: If the channel at wavelength $\lambda_{k}$ is dropped at node NODE_DEST $i$, the channel power at the receiver needs to be greater than the receiver sensitivity $\left(P_{\text {minr }}\right)$ to enable a low BER of the detected signal

$$
\begin{aligned}
& P_{i, k}+G_{i}-\alpha L_{i}-\beta 2_{\text {NODE_DEST }_{i}} \geq P_{\mathrm{minr}} \\
& \forall \lambda_{k} \in \mathrm{DROP}_{\text {NODE_DEST }_{i}} .
\end{aligned}
$$

The received power of a channel also needs to be within the dynamic range of the receiver

$$
\begin{array}{r}
P_{i, k}+G_{i}-\alpha L_{i}-\beta 2_{\text {NODE_DEST }} \leq P_{\text {minr }}+\text { DR } \\
\forall \lambda_{k} \in \text { DROP }_{\text {NODE_DEST }_{i}} .
\end{array}
$$

h) Constraints for in-band crosstalk: If wavelength $\lambda_{k}$ is both added and dropped at node NODE_DEST $i$, the in-band crosstalk from the drop to add channels at $\lambda_{k}$ is required to be below $-25 \mathrm{~dB}$ to achieve a power penalty below $1 \mathrm{~dB}$ [15] as shown in (21), shown at the bottom of the page. Similarly, the in-band crosstalk from the add to drop channels at $\lambda_{k}$ is also required to be below $-25 \mathrm{~dB}$, as shown in (22), shown at the bottom of the page.

i) Constraint for total ring gain: In order to prevent lasing and accumulation of ASE noise in the ring, the total gain is required to be less than the total loss by a margin, as follows:

$$
\left(\sum_{i=1}^{L} \alpha L_{i}+\sum_{j=1}^{N} \beta 1_{j}\right)-\sum_{i=1}^{L} G_{i} \geq \text { MARGIN. }
$$

Objective Function: The objective function of the amplifier placement problem is to minimize the total number of amplifiers in the ring network

$$
\text { Minimize } \sum_{i=1}^{L} n_{i} .
$$

\section{B. Solution Method}

The problem described in Section III-A contains highly nonlinear constraints (Constraints (4) and (14) for Method $1 \mathrm{~A}$ and Constraints (4b) and (14b) for Method 1B). The other constraints and the objective function are linear. Due to the presence of both integer and continuous variables, the amplifier placement problem is extremely difficult to solve and is highly computation intensive. The problem belongs to the class of MINLP problems, and in general, this class of problem is classified as nondeterministic polynomial (NP)-hard [16]. Several algorithms have been proposed to solve such problems and can be found in [17]. Software for this particularly difficult kind of optimization has typically followed two algorithms [18].

1) Outer approximation/generalized benders decomposition: These algorithms alternate between solving an MILP master problem and nonlinear programming (NLP) subproblems.

2) Branch and bound $(B \& B)$ : B \& B methods have been successfully used for MILP and can be extended to the nonlinear case.

The MINLP problem described in Section III-A was programmed in the AMPL modeling language [19] and solved by the MINLP solver from the NEOS server [20]. The MINLP solver implements the B \& B method using a depth-first search [21]. The resulting NLP relaxations are then solved using filterSQP [22].

There are some limitations in using the MINLP solver for solving the amplifier placement problem, and they are discussed hereafter.

$$
\begin{aligned}
& \left(P_{i, k}+G_{i}-\alpha L_{i}+I X 1_{\mathrm{NODE}_{\text {DEEST }}}\right)-\left(P_{\mathrm{NODE}_{i} \mathrm{XEST}_{i}, k}-\beta 3_{\mathrm{NODE}_{\text {DEST }}}\right) \leq-25 \\
& \forall \lambda_{k} \in\left(\mathrm{DROP}_{\mathrm{NODE}_{\text {DEST }}} \cup \mathrm{ADD}_{\mathrm{NODE}_{\text {_DEST }}}\right) .
\end{aligned}
$$

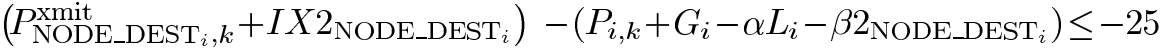

$$
\begin{aligned}
& \forall \lambda_{k} \in\left(\mathrm{DROP}_{\mathrm{NODE}_{\mathrm{NEST}}} \cup \mathrm{ADD}_{\mathrm{NODE}_{\text {DEST }}}\right) \text {. }
\end{aligned}
$$




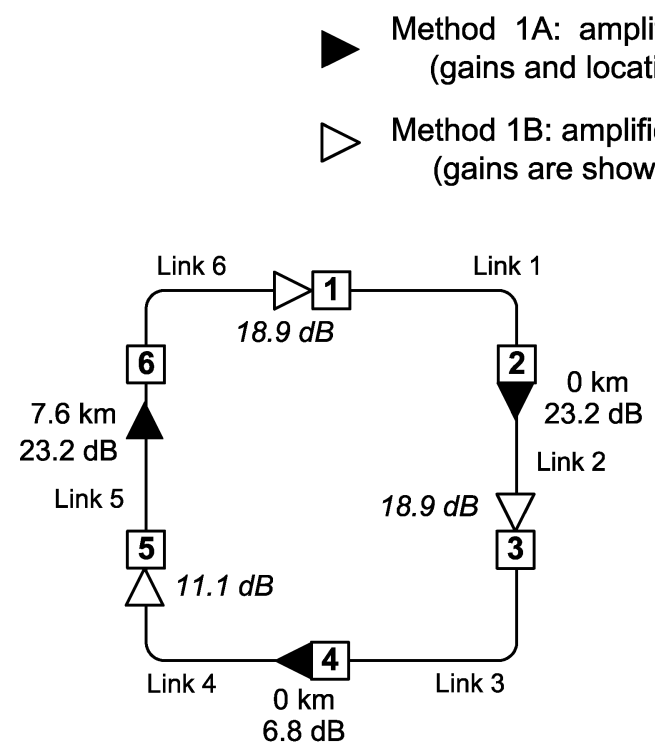

(a)

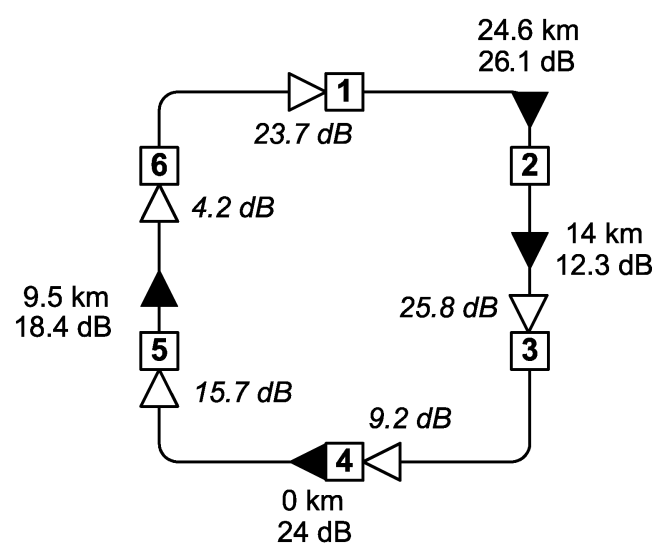

(b)

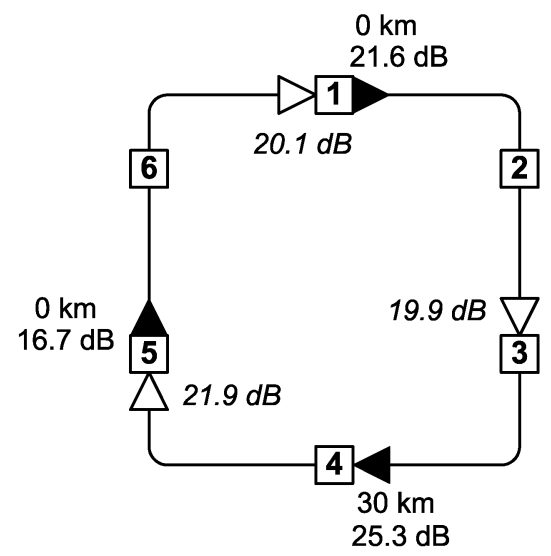

(c)

Fig. 4. Amplifier placement solutions for six-node logical-meshed rings with different link lengths using Method 1: (a) 10-km node spacing, (b) 30-km node spacing, (c) link lengths: $1-2-3-4-5-6=10-20-30-20-10-30 \mathrm{~km}$.

1) Local minima: A solution from the MINLP solver may correspond to a local minima for the objective function due to the mixed-integer nonlinear nature of the problem. The MINLP solver using the B \& B algorithm is more robust than the outer approximation or benders decomposition which usually cut away large parts of the feasible region [23].

2) Feasible point generation: When the starting point is infeasible, the MINLP solver may not be able to find a feasible point in the search space. In this case, the algorithm is said to be in Phase I and aims to converge to a Kuhn-Tucker point of a feasibility problem [24]. The solution of the feasibility problem indicates which constraints are causing the problem to be infeasible, and the user can modify the problem accordingly. However, finding a feasible point becomes harder as the ring size increases.

\section{Numerical Results and Discussion}

For each ring configuration, the solution from the MINLP solver provides the number of amplifiers in the ring, the gain and location of each amplifier, and the transmitted power of each channel at the output of each node.

Amplifier placement solutions for a six-node ring with different node spacings are shown in Fig. 4. For networks with a short node spacing of $10 \mathrm{~km}$, as shown in Fig. 4(a), the solutions from both methods provide fewer amplifiers than the number of links. As the node spacing increases to $30 \mathrm{~km}$, as shown in Fig. 4(b), more amplifiers are needed, and the solutions converge to one amplifier required per link to satisfy all constraints. In all amplifier placement cases shown in Fig. 4, solutions using Method 1A require equal or fewer amplifiers than those using Method 1B. This is because by placing the amplifier anywhere on a link, the signal power can be at the lowest allowable level before being amplified. On the other hand, when amplifiers are located at the end of links, the signal power at the beginning of a link needs to be high enough to travel the whole link before being amplified; this may require an additional amplifier at the previous link. Consequently, the solution by Method 1B may require more amplifiers than that by Method 1A.

The number of amplifiers for different ring networks are summarized in Table IV. The results in Table IV confirm that solu- 
TABLE IV

NUMBER OF AMPLIFIERS IN DIFFERENT RING NETWORKS USING METHOD 1

\begin{tabular}{c|c|c}
\hline \multirow{2}{*}{ Ring Network } & \multicolumn{2}{|c}{ Number of Amplifiers } \\
\cline { 2 - 3 } & $\begin{array}{c}\text { Method 1A } \\
\text { (amplifier placed } \\
\text { anywhere on link) }\end{array}$ & $\begin{array}{c}\text { Method 1B } \\
\text { (amplifier placed } \\
\text { at end of link) }\end{array}$ \\
\hline 6-node (10-km node spacing) & 3 & 3 \\
\hline 6-node (30-km node spacing) & 4 & 5 \\
\hline 6-node (Link lengths: 1-2-3-4-5-6 & 3 & 3 \\
\hline 10-20-30-20-10-30 km) & 6 & 6 \\
\hline 10-node (10-km node spacing) & 9 & 8 \\
\hline 10-node (30-km node spacing) & 7 & \\
\hline 8-9-10=10-20-30-10-20-20-30-20- & & \\
\hline $10-30 \mathrm{~km})$ & & \\
\hline
\end{tabular}

tions using Method $1 \mathrm{~A}$ have equal or fewer amplifiers than those using Method 1B. For practical considerations, placing amplifiers at the end of the links is more preferable than placing amplifiers anywhere on the links. Placing amplifiers at the end of the links simplifies the ring network by placing active components next to the OADM, which also enhances network maintainability and reduces cost. Moreover, the amplifiers at the end of the links can serve as preamplifiers to enhance the signal quality before the receiver.

Method 1 can be applied to rings, mesh, or logical star networks to provide a solution to effectively place amplifiers in the networks as long as we can keep the requirement of one amplifier per link. However, for large networks, a feasible amplifier placement solution may not be found because amplifiers are driven deep into the saturation region due to the presence of many wavelength channels and, hence, they could not provide enough gain to satisfy all constraints. Consequently, more than one amplifier may be required on each link. Moreover, as the network gets bigger, it becomes increasingly difficult for the MINLP solver to find a feasible solution due to a large number of variables and nonlinear constraints. Commercial and trial software for solving MINLP problems are still limited in their ability to solve large and highly nonlinear problems. Approaches using heuristic search methods such as simulated annealing, tabu search, and genetic algorithms have also been extensively studied to tackle this kind of difficult problems [25].

\section{AmPlifier Placement IN Single-Fiber WDM Rings: MILP METHOD (METHOD 2)}

The amplifier placement Method 1 presented in Section III requires solving the MINLP problem, which contains two nonlinear Constraints (4) and (14) for Method 1A and two nonlinear Constraints (4b) and (14b) for Method 1B. These nonlinear constraints make it very difficult to solve the problem optimally. Therefore, it is important that the nonlinear constraints can be approximated by linear constraints so that more efficient solutions can be obtained.

In this section, the amplifier placement Method 2 based on the integer linear programming technique is presented. The nonlinear constraints in Method 1 are linearly approximated. The result is an MILP problem. Similarly to Method 1, Method 2 is referred to as either Method 2A or Method 2B, depending on the amplifier location. Method 2A allows the amplifiers to be placed anywhere on the links, and Method 2B allows the amplifiers only to be placed at the end of the links.

\section{A. Linear Approximation of Constraints (4) and (4b)}

Constraint (4) in Method 1A can be rewritten in the form

$$
y=10 \log _{10}\left(\sum_{k=1}^{W+1} 10^{\frac{x_{k}}{10}}\right)
$$

where

$$
\begin{aligned}
& y \quad \text { is the total power at the beginning of link } \\
& i\left(y=P_{i}^{\text {tot }}+\alpha K_{i}\right) \text {; } \\
& x_{k}(k=1, \ldots, W) \text { is the transmitted power of the channel at } \\
& \text { wavelength } \lambda_{k}\left(x_{k}=P_{i, k}\right) \text {; } \\
& x_{W+1} \quad \text { is the total ASE noise power from }
\end{aligned}
$$

The least-squares fit (LSF) [26] is used to provide a best-fit approximation of (25) by the linear function of the form

$$
y=\sum_{k=1}^{W+1} h_{k} x_{k}+h_{W+2} .
$$

There are several notations used in the problem statement.

1) $M$ is the number of data samples taken for each $x_{k}$ term in (25) for the linear approximation.

2) $\boldsymbol{x}^{j}=\left[\begin{array}{lllll}x_{1}^{j} & x_{2}^{j} & \ldots & x_{w+1}^{j} & 1\end{array}\right]^{T}$ for $j=1, \ldots, M ; \boldsymbol{x}^{j}$ is a $(W+2) \times 1$ vector. Each element $x_{k}^{j}$ in vector $\boldsymbol{x}^{j}$, $\mathrm{k}=1, \ldots,(W+1)$ corresponds to one data sample of the $x_{k}$ term in (25).

3) $\boldsymbol{h}=\left[\begin{array}{lllll}h_{1} & h_{2} & \ldots & h_{W+1} & h_{W+2}\end{array}\right]^{T}$, and $\boldsymbol{h}$ is a $(W+2) \times 1$ vector.

4) $y^{j}$ is a function of $x_{k}^{j}$ and is given as

$$
y^{j}=10 \log _{10}\left(\sum_{k=1}^{W+1} 10^{\frac{x_{k}^{j}}{10}}\right)
$$

where $y^{j}$ represents the value $y$ in (25), corresponding to each set of data samples $j=1, \ldots, M$.

The LSF requires that to linearly approximate function $y$ in (25), the following function $C$ needs to be minimized with respect to $\boldsymbol{h}$, as follows:

$$
C=\sum_{j=1}^{M}\left\|\boldsymbol{h}^{T} x^{j}-y^{j}\right\|^{2}
$$

where $\boldsymbol{h}^{T}$ is the transpose of vector $\boldsymbol{h}$.

Equation (27) can be rewritten as

$$
C=\boldsymbol{h}^{T} \boldsymbol{D} \boldsymbol{h}-2 \boldsymbol{E} \boldsymbol{h}+\sum_{j=1}^{M}\left(y^{j}\right)^{2}
$$


where $\boldsymbol{D}=\sum_{j=1}^{M}\left[\boldsymbol{x}^{j}\left(\boldsymbol{x}^{j}\right)^{T}\right]$, and $\boldsymbol{E}=\sum_{j=1}^{M}\left[y^{j}\left(\boldsymbol{x}^{j}\right)^{T}\right]$. It can be easily seen that $C$ is minimized when $\boldsymbol{h}=D^{-1} \boldsymbol{E}$, where $D^{-1}$ is the inverse of $D$. Therefore

$$
\boldsymbol{h}=\left[\sum_{j=1}^{M}\left(\boldsymbol{x}^{j}\left(\boldsymbol{x}^{j}\right)^{T}\right)\right]^{-1} \cdot\left[\sum_{j=1}^{M}\left(y^{j}\left(\boldsymbol{x}^{j}\right)^{T}\right)\right] .
$$

The vector $\boldsymbol{h}$ in (29) is calculated using MATLAB. In the implementation, $M=10000$ data samples are taken for each $x_{k}$ term in (25). From the device parameters shown in Table I, $P_{i, k}$ varies from -30 to $-5 \mathrm{dBm}$ and $P_{\mathrm{SRC}_{i}}^{\text {totase }}$ varies from -50 to 0 $\mathrm{dBm}$. Therefore, the sample values of $P_{i, k}$ are taken randomly from -30 to -5 assuming a uniform distribution and the sample values of $P_{\mathrm{SRC}_{i}}^{\mathrm{tot} \text { ase }}$ are taken randomly from -50 to 0 also assuming a uniform distribution.

The nonlinear Constraint (4) in Method 1A and nonlinear Constraint (4b) in Method 1B can now be approximated by the linear Constraint (30) for Method 2A and linear Constraint (30b) for Method 2B, respectively, as follows:

$$
\begin{aligned}
P_{i}^{\mathrm{tot}}= & \sum_{k=1}^{W}\left(h_{k} P_{i, k}\right)+h_{W+1} \\
& \times\left(P_{\mathrm{SRC}_{i}}^{\mathrm{tot}}-\beta 1_{\mathrm{NODE}_{\text {SRC }}}-A+B\right) \\
& +h_{W+2}-\alpha K_{i} \\
P_{i}^{\mathrm{tot}}= & \sum_{k=1}^{W}\left(h_{k} P_{i, k}\right)+h_{W+1} \\
& \times\left(P_{\mathrm{SRC}_{i}}^{\mathrm{tot} \text { ase }}-\beta 1_{\mathrm{NODE}_{\mathrm{SRC}}}-A+B\right) \\
& +h_{W+2}-\alpha L_{i}
\end{aligned}
$$

where $h_{k}(k=1, \ldots, W), h_{W+1}$, and $h_{W+2}$ are derived from the linear approximation described in (29).

\section{B. Linear Approximation of Constraints (14) and (14b)}

If the amplifier gain is assumed to be much greater than 1 $\left(10^{G_{i} / 10} \gg 1\right)$, Constraint (14) can be approximated as

$$
\begin{aligned}
P_{i}^{\text {tot_ase }} \approx & G_{i}-\alpha L_{i}+10 \log _{10} \\
& \times\left[10^{\frac{\left(\alpha K_{i}+A\right)}{10}}+10 \frac{\left(P_{\mathrm{SRC}_{i}}^{\text {tot_ase }}-\beta 1_{\mathrm{NODE}-\mathrm{SRC}_{i}}\right)}{10}\right] .
\end{aligned}
$$

The third term of $P_{i}^{\text {tot_ase }}$ in (31) has the same format as (25) described in Section IV-A and is given as

$$
y=10 \log _{10}\left(10^{\frac{x_{1}}{10}}+10^{\frac{x_{2}}{10}}\right)
$$

where $x_{1}=\alpha K_{i}+A$ and $x_{2}=P_{\mathrm{SRC}_{i}}^{\text {tot_ase }}-\beta 1_{\text {NODE_SRC }_{i}}$. We try to find the coefficient vector $\boldsymbol{h}=\left[\begin{array}{lll}h_{1} & h_{2} & h_{3}\end{array}\right]^{T}$ so that $y$ in (32) can be linearly approximated as

$$
y=h_{1} x_{1}+h_{2} x_{2}+h_{3} .
$$

Equations (31)-(33) are for Method 2A, where amplifiers can be placed anywhere on the links. For Method 2B, where amplifiers can only be placed at the end of the links, $K_{i}=L_{i}$ and (31),
(32), and (33) are modified to become (31b), (32b), and (33b), respectively

$$
\begin{aligned}
P_{i}^{\text {tot_ase }}= & G_{i}-\alpha L_{i}+10 \log _{10} \\
& \times\left[10^{\frac{\left(\alpha L_{i}+A\right)}{10}}+10 \frac{\left(P_{\mathrm{SRC}_{i}}^{\text {tot_ase }}-\beta 1_{\mathrm{NODE}_{\text {SRC }}}\right)}{10}\right] \\
y= & 10 \log _{10}\left(10^{\frac{x_{1}}{10}}+F\right) \\
y= & h_{1} x_{1}+h_{2}
\end{aligned}
$$

where $x_{1}=P_{\mathrm{SRC}_{i}}^{\text {tot_ase }}-\beta 1_{\mathrm{NODE}_{\mathrm{SRC}}}$ and $F=10^{\left(\alpha L_{i}+A\right) / 10}$. Similarly to the approach used in approximating Constraint (4), LSF is used to calculate $h_{1}, h_{2}$, and $h_{3}$ in (33) and $h_{1}$ and $h_{2}$ in (33b).

The nonlinear Constraint (14) in Method 1A and the nonlinear Constraint (14b) in Method 1B can now be approximated by the linear Constraint (34) for Method 2A and linear Constraint (34b) for Method 2B, respectively, as follows:

$$
\begin{aligned}
P_{i}^{\text {tot_ase }}= & G_{i}-\alpha L_{i}+h_{1}\left(\alpha K_{i}+A\right) \\
& +h_{2}\left(P_{\mathrm{SRC}_{i}}^{\text {tot_ase }}-\beta 1_{\mathrm{NODE} \_\mathrm{SRC}_{i}}\right)+h_{3} \\
P_{i}^{\text {tot_ase }}= & G_{i}-\alpha L_{i}+h_{1}\left(P_{\mathrm{SRC}_{i}}^{\text {totase }}-\beta 1_{\left.\mathrm{NODE}_{\mathrm{SRC}_{i}}\right)+h_{2}}\right.
\end{aligned}
$$

where $h_{1}, h_{2}$, and $h_{3}$ in Constraint (34) and $h_{1}$ and $h_{2}$ in Constraint (34b) are derived from the linear approximation described in (29).

\section{Numerical Results and Discussion}

The amplifier placement problem used in Method 2A consists of the same constraints and objective function as that in Method 1A described in Section III-A except that Constraints (4) and (14) are substituted by Constraints (30) and (34), respectively. The problem in Method 2B is similar to that in Method 1B but has Constraints (30b) and (34b) instead of Constraints (4b) and (14b), respectively. The amplifier placement problem used in Methods 2A and 2B contains only linear constraints and objective function and is classified as MILP problems. The MILP problem can now be optimally solved.

The MILP problem was programmed in the AMPL modeling language and solved using a commercial MILP solver, XPRESS from Dash Associates [27]. XPRESS uses the B \& B algorithm [28] to handle the integrality constraints, thereby significantly reducing the exponential search space.

The amplifier placement solution for six-node rings using Method 2 is shown in Fig. 5 for different link lengths. The results for six-node rings shown in Fig. 5 using Method 2 generally require more amplifiers than the results shown in Fig. 4 using Method 1. This is because the MILP problem uses linear approximations of the nonlinear constraints in the MINLP problem, which results in a loss of accuracy. The approximate linear constraints are stricter than the exact nonlinear constraints; therefore, the solutions from the MILP problem may require more amplifiers than those from the MINLP problem.

Table V summarizes the number of amplifiers and CPU time taken for different rings from the solutions using Method 1B and Method 2B. For six-node rings, the results between the two 


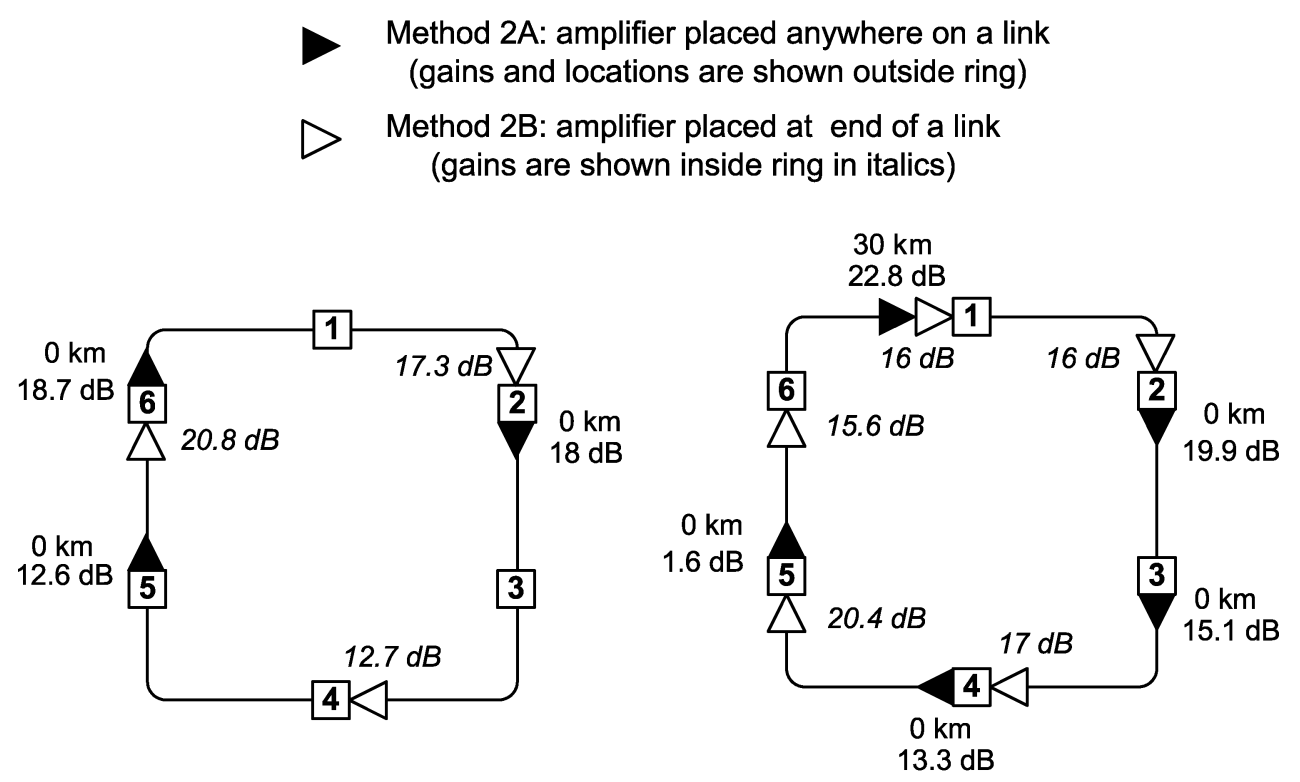

(a)

(b)

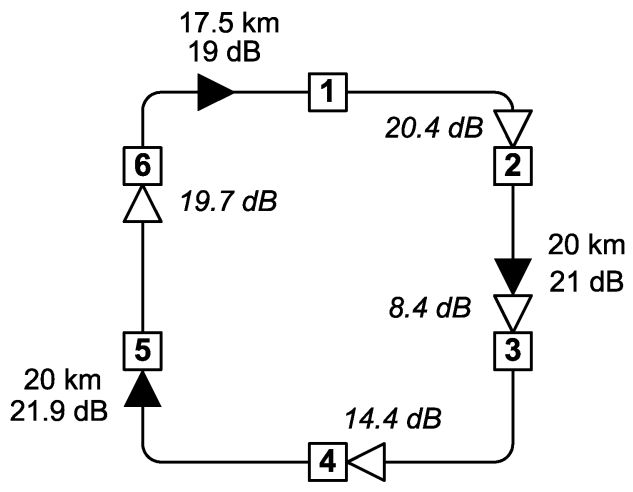

(c)

Fig. 5. Amplifier placement solution for six-node rings using Method 2: (a) 10-km node spacing, (b) $30-\mathrm{km}$ node spacing, (c) link lengths: $1-2-3-4-5-6=$ $10-20-30-20-10-30 \mathrm{~km}$.

TABLE V

NUMBER OF AMPLIFIERS AND CPU TIME TAKEN FOR DIFFERENT RINGS USING METHOD 1B AND METHOD 2B

\begin{tabular}{|c|c|c|c|c|}
\hline \multirow{2}{*}{ Ring Network } & \multicolumn{2}{|c|}{$\begin{array}{l}\text { Number of } \\
\text { Amplifiers }\end{array}$} & \multicolumn{2}{|c|}{$\begin{array}{l}\text { CPU Time } \\
\text { (minutes) }\end{array}$} \\
\hline & $\begin{array}{c}\text { Method } \\
\text { 1B }\end{array}$ & $\begin{array}{c}\text { Method } \\
\text { 2B }\end{array}$ & $\begin{array}{l}\text { Method } \\
\text { 1B }\end{array}$ & $\begin{array}{c}\text { Method } \\
\text { 2B }\end{array}$ \\
\hline 6-node (10-km node spacing) & 3 & 3 & 4 & 1 \\
\hline 6-node (30-km node spacing) & 5 & 5 & 1 & 1 \\
\hline $\begin{array}{l}\text { 6-node (Link length: } 1-2-3-4-5-6 \\
=10-20-30-20-10-30-\mathrm{km})\end{array}$ & 3 & 4 & 1 & 1 \\
\hline 10-node (10-km node spacing) & 6 & 9 & 26 & 1 \\
\hline 10-node (30-km node spacing) & 10 & Infeasible & 3 & Infeasible \\
\hline $\begin{array}{l}\text { 10-node (Link length: } 1-2-3-4-5- \\
6-7-8-9-10=10-20-30-10-20-20- \\
30-20-10-30 \mathrm{~km})\end{array}$ & 8 & 10 & 11 & 1 \\
\hline
\end{tabular}

solutions are very similar. However, for ten-node rings, Method 1B gives fewer amplifiers than Method 2B. The reason is that for small networks, the linear approximation is very close to the actual function so the two sets of constraints in the two problems are not much different. Consequently, the results from the two methods are similar to each other. For large networks with a large number of variables and constraints, the linear approximation is further away from the actual function, which makes constraints in the MILP problem much stricter than those in the MINLP problem. Therefore, more amplifiers may be required in the solution of the MILP problem compared with that of the MINLP problem. Moreover, the stricter constraints may make the MILP problem infeasible. On the other hand, the MILP problem generally takes less time to solve than the MINLP problem.

The amplifier placement Method 2 using the MILP problem produces an optimal number of amplifiers in the networks since the problem is linear. However, due to the approximation of the nonlinear constraints, the result from solving the MILP problem is an overengineered solution that has more amplifiers than necessary to operate the networks. Both amplifier placement Methods 1 and 2 can be used in conjunction to optimally place amplifiers in a ring network. Method 2 can be used first to achieve an approximate solution with fast computation time. 
Method 1 can then be employed to provide an exact solution to the problem.

\section{AmPLifier Placement IN SELF-HeAling WDM RingS}

In Sections III and IV, two methods to provide an efficient number of amplifiers to operate the networks based on integer programming techniques were presented. However, these methods only provide amplifier placement solutions for single-fiber rings. Self-healing WDM rings with multiple fibers based on synchronous optical network/synchronous digital hierarchy (SONET/SDH) self-healing rings have been proposed to facilitate network survivability [3], [4], [29], [30]. In self-healing rings, the networks need to reconfigure themselves to recover from node and/or link failures, and therefore, the amplifier placement solution needs to make sure that under a normal or any failure conditions, the network is still operational.

\section{A. Network Structure}

A typical self-healing WDM ring proposed in [4] and [30] is chosen for the illustration of the amplifier placement method. The ring is a logical-meshed unidirectional self-healing WDM ring with passive protection and is shown in Fig. 6(a) for a network under a normal condition and Fig. 6(b) for a network under a link failure condition. The ring consists of one working fiber and one protection fiber. The protection and working fibers are assumed to have equal lengths. The protection fiber is passive and only carries signals in the event of a link failure in the network. Protection in the self-healing ring is achieved via the use of two optical switches (OSW1 and OSW2) located at each node. Note that this self-healing ring architecture only provides protection against a single link failure.

In this study, we also assume that at most one amplifier is required on each link to compensate for the losses. The amplifier is assumed to be placed at the node, specifically after OSW1 on the working fiber and after OSW2 on the protection fiber, as shown in Fig. 6. With this configuration, the amplifier at the node can still be used even when there is a cable failure between that node and the preceding node due to the loop-back of the OSWs. For example, the amplifier at node 1 in Fig. 6(b) can be used even when there is a link failure between nodes 1 and 4 .

Each node has an OADM, two OSWs, and amplifier(s) as shown in Fig. 6. The OADM at each node is capable of adding/dropping $(N-1)$ different wavelengths to/from other nodes and is assumed to have the MUX/DEMUX configuration as in [11].

\section{B. Iterative Method}

The amplifier placement method for self-healing WDM rings is referred to as the iterative method and involves formulating the amplifier placement problem and iteratively solving for the optimal number of amplifiers in a self-healing ring under a normal and single-link-failure conditions. The amplifier placement problem formulation for each ring under a normal or a link-failure condition is similar to that described in Section III-A and will not be repeated here. However, since this is a self-healing ring with passive protection, constraints are

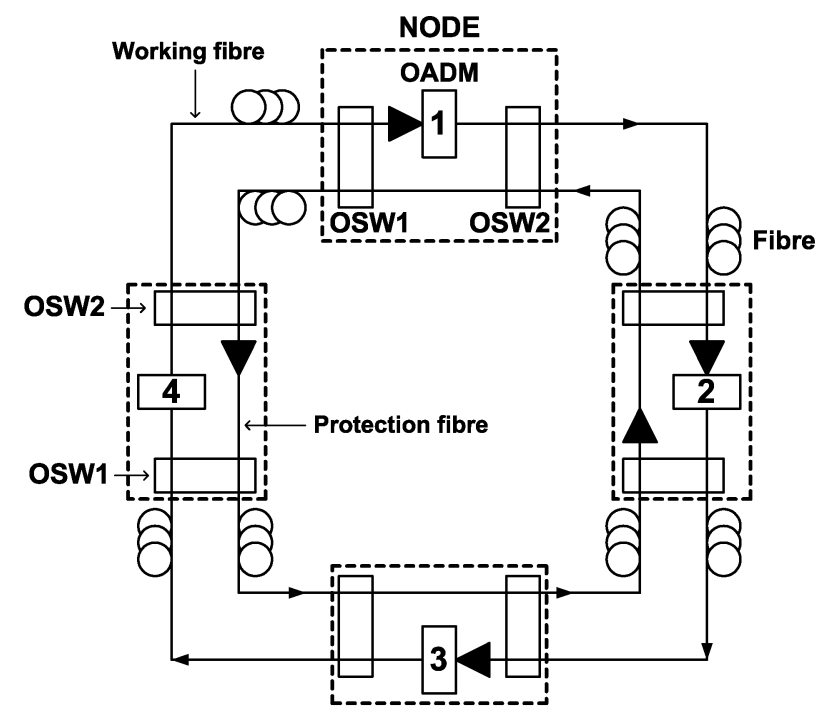

(a)

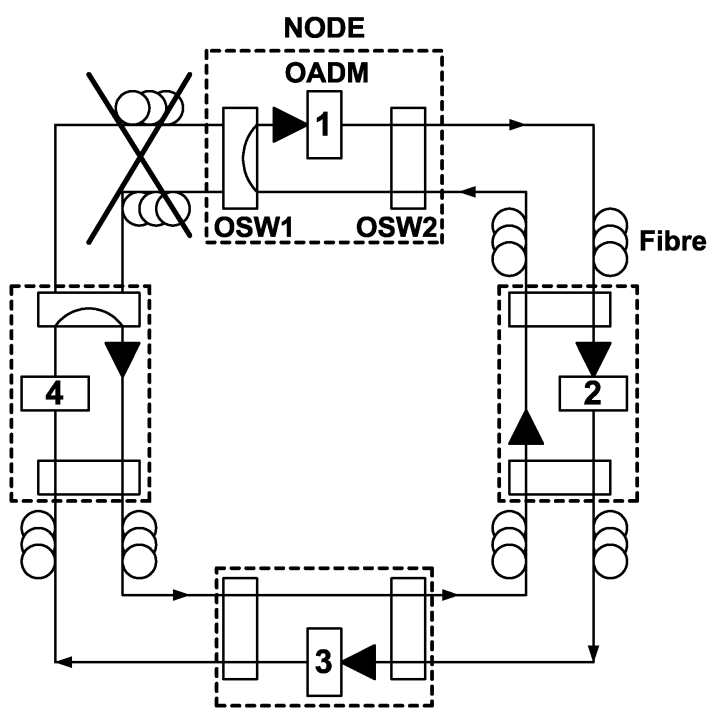

(b)

Fig. 6. Four-node logical-meshed unidirectional self-healing WDM ring with passive protection: (a) ring under a normal condition and (b) ring under a link failure between node 1 and node 4 .

different for the working and protection fibers. The design variables of the problem are the number of amplifiers, the location of each amplifier, and the gain of each amplifier. Unlike the study in Sections III and IV, in this section, channel-transmitted powers at each node are fixed to simplify the network management and control system.

The iterative method for optimal amplifier placement in selfhealing WDM rings is illustrated in Fig. 7. The iterative method uses an MINLP solver to solve the amplifier placement problem for a ring network under a link failure. Then, the ring is assumed to be under a different link failure; the new problem is to optimize the amplifier placement in the new ring configuration taking into account the amplifier placement solution from the previous failure scenario. This process is repeated until all link failures in the ring network are taken into account. Then, the amplifier placement problem is solved for the ring under a normal condition taking into account the previous solution. 


\section{Step 1}

- Choose the shortest link in the self-healing ring. Assume that there is a failure on that link.

- Optimize the number and placement of amplifiers for the ring under this link failure. The solution contains the largest number of amplifiers among all link failure scenarios since the ring under this link failure has the longest protection path.

- Create an amplifier placement table for the self-healing ring.

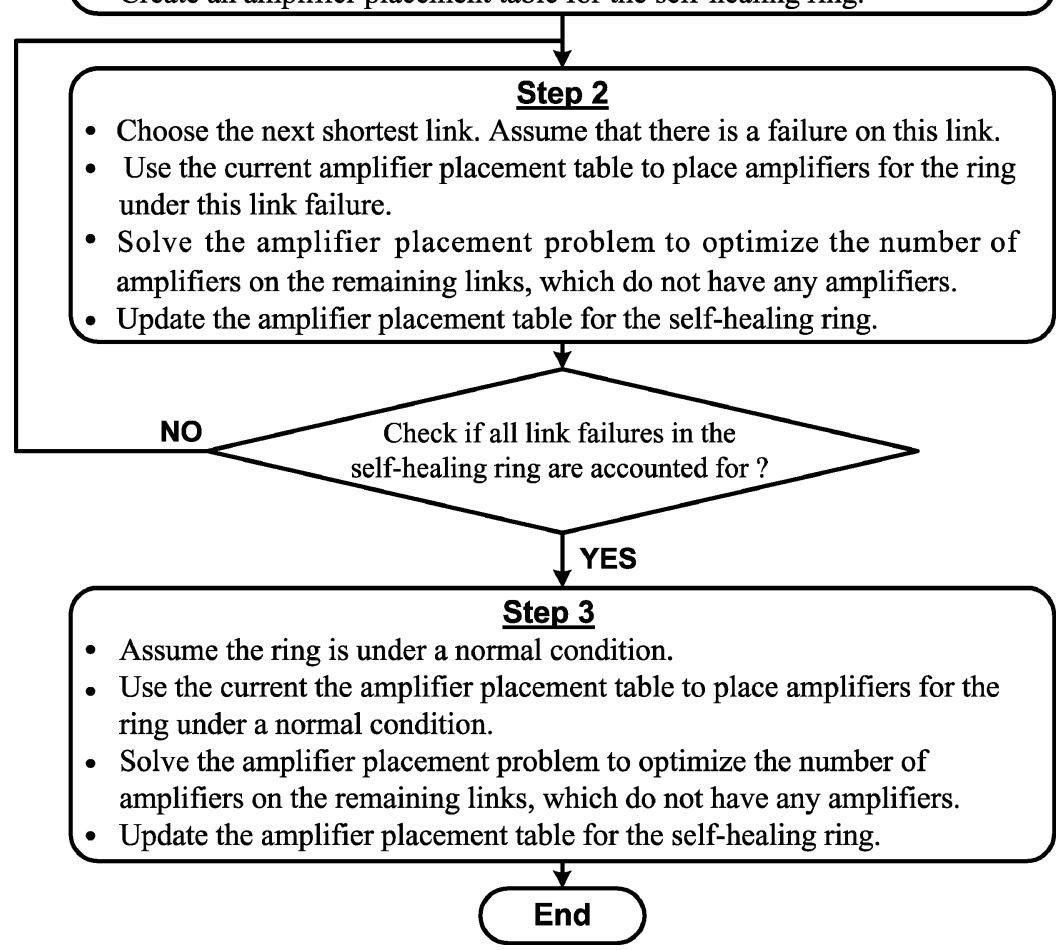

Fig. 7. Iterative method for amplifier placement in self-healing WDM rings.

The final amplifier placement solution guarantees that the network is functional under normal or any link-failure scenarios. This is because the final solution contains all the amplifiers required to operate each ring configuration under normal or any cable failure conditions. If there are more amplifiers than required for each ring configuration, the gain of the additional amplifiers can be set to $0 \mathrm{~dB}$, and those amplifiers do not affect any of the ring constraints. To minimize the number of amplifiers in the self-healing ring, the order of link failure scenarios with increasing link lengths is chosen for the iterative method.

\section{Numerical Results and Discussion}

Fig. 8 shows the amplifier placement solution using the iterative method for a six-node self-healing ring with link 1,2,3, 4,5 , and 6 having a length of $10,20,30,20,10$, and $30 \mathrm{~km}$, respectively (denoting as link lengths: $1-2-3-4-5-6=$ $10-20-30-20-10-30 \mathrm{~km}$ ). The solution requires eight amplifiers to operate the self-healing ring. If one amplifier is used at each node in both the working and protection fibers as proposed in [3] and [4], 12 amplifiers are required to operate the ring network shown in Fig. 8. The solution using the iterative method is able to save four amplifiers for this ring.

Like the amplifier placement problem for single-fiber rings presented in Section III, in this chapter, amplifier gains are design variables. They are obtained from solving the amplifier

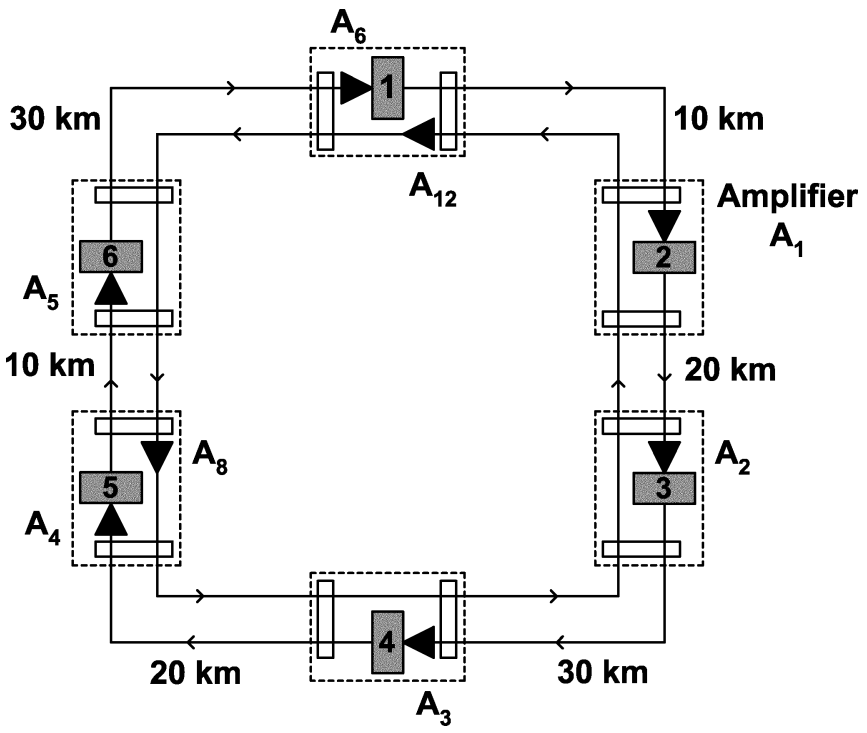

Fig. 8. Amplifier placement solution for a six-node self-healing ring with link lengths: $1-2-3-4-5-6=10-20-30-20-10-30 \mathrm{~km}$.

placement problem. Under different link failures, each amplifier may have a different gain. The network management and control system needs to identify the failure in the network and then inform each node to configure its amplifiers accordingly. The 
TABLE VI

Minimum Amplifier Gains for a SiX-Node SELF-HeAling Ring With Link Lengths

$1-2-3-4-5-6=10-20-30-20-10-30 \mathrm{~km}$

UNDER DIFFERENT LINK FAILURES

\begin{tabular}{c|c|c|c|c|c|c|c}
\hline \multirow{2}{*}{$\begin{array}{c}\text { Amp. } \\
\text { No. }\end{array}$} & \multicolumn{7}{|c}{ Amplifier Gain (dB) } \\
\cline { 2 - 8 } & $\begin{array}{c}\text { No } \\
\text { failure }\end{array}$ & $\begin{array}{c}\text { Failure } \\
\text { between } \\
\mathbf{1} \& \mathbf{2}\end{array}$ & $\begin{array}{c}\text { Failure } \\
\text { between } \\
\mathbf{2} \text { \& 3 }\end{array}$ & $\begin{array}{c}\text { Failure } \\
\text { between } \\
\mathbf{3 ~ \& ~ 4 ~}\end{array}$ & $\begin{array}{c}\text { Failure } \\
\text { between } \\
\mathbf{4 ~ \& ~ 5 ~}\end{array}$ & $\begin{array}{c}\text { Failure } \\
\text { between } \\
\mathbf{5} \& \mathbf{6}\end{array}$ & $\begin{array}{c}\text { Failure } \\
\text { between } \\
\mathbf{6} \text { \& 1 }\end{array}$ \\
\hline $\mathbf{A}_{\mathbf{1}}$ & 7.4 & 6 & 7.2 & 7.5 & 9.6 & 8.3 & 7.6 \\
\hline $\mathbf{A}_{\mathbf{2}}$ & 13.4 & 13.5 & 4 & 13.4 & 14.2 & 13.2 & 13.2 \\
\hline $\mathbf{A}_{\mathbf{3}}$ & 12.1 & 11.9 & 13 & 0 & 14.8 & 12.5 & 12.1 \\
\hline $\mathbf{A}_{\mathbf{4}}$ & 11 & 11 & 10 & 10.7 & 0 & 10.9 & 10.9 \\
\hline $\mathbf{A}_{\mathbf{5}}$ & 9.7 & 9.6 & 9.9 & 9.9 & 5.9 & 0.6 & 9.6 \\
\hline $\mathbf{A}_{\mathbf{6}}$ & 14.4 & 14.4 & 13.9 & 14.3 & 16.7 & 13.5 & 0 \\
\hline $\mathbf{A}_{\mathbf{8}}$ & Unused & 10.9 & 12.3 & 8.7 & 8.7 & 15.8 & 17.6 \\
\hline $\mathbf{A}_{\mathbf{1 2}}$ & Unused & 15.4 & 18 & 20.5 & 24.8 & 18.6 & 13.7 \\
\hline & & & & & & & \\
\hline
\end{tabular}

amplifier gain for each failure condition can be calculated beforehand using the proposed method and is stored at each node. Once the failure is identified, the node needs to look up its amplifier gain table and adjust the pump power for the amplifiers.

Minimizing the amplifier gain helps reduce the power consumption at each node. In order to minimize the gain of each individual amplifier in the self-healing ring for different failure scenarios, the problem presented in Section III-A is slightly modified. The number of amplifiers on link $i\left(n_{i}\right)$ now becomes a parameter. $n_{i}$ is given from the solution using the iterative method. The new objective function is to minimize the total gain of amplifiers in the network and is given as

$$
\text { Minimize } \sum_{i=1}^{L} G_{i} \text {. }
$$

The minimum amplifier gains under different link failures are shown in Table VI. The results show that under some failure conditions, some amplifier gains are set to $0 \mathrm{~dB}$.

\section{Effect of Node Failure}

The iterative method provides an amplifier placement solution for a self-healing ring to ensure a working network under a normal condition or a single link failure between two adjacent nodes. When a node fails, the solution still guarantees that all network and device constraints are satisfied for the ring under the node failure.

Consider the amplifier placement solution for the six-node self-healing ring shown in Fig. 8, which consists of eight amplifiers. When there is a failure at node 1, the ring is shown in Fig. 9. In the self-healing ring under a failure at node 1 , the OSWs at node 2 and node 6 adjacent to the failed node 1 are looped back to provide a protection path for the signals from node 6 to node 2 via the protection fiber, following the approach proposed in [4]. The amplifiers on link 6 and on link 12 are no longer used for the new ring configuration. However, the amplifier placement solution for the self-healing ring already ensures the ring is operational under a link failure between nodes 6 and 1 or between nodes 1 and 2 . Therefore, with the remaining six amplifiers, it is still guaranteed that all network and device constraints are met

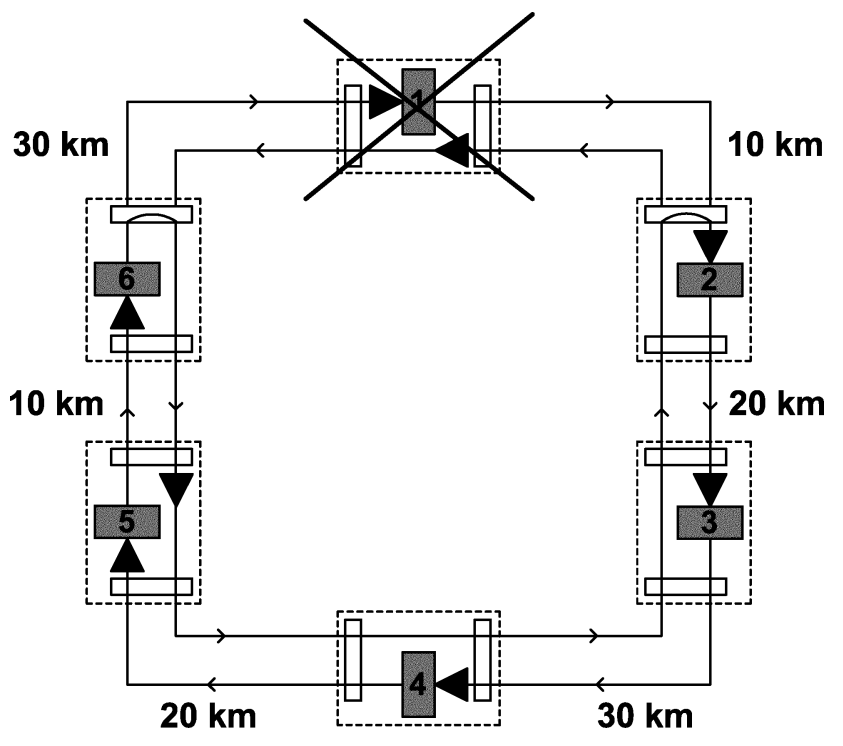

Fig. 9. Six-node self-healing ring with link lengths $1-2-3-4-5-6=$ $10-20-30-20-10-30 \mathrm{~km}$ under a failure at node 1 .

for the ring network containing the remaining nodes 2, 3, 4, 5, and 6.

The minimum amplifier gains for the six-node self-healing ring with link lengths $1-2-3-4-5-6=10-20-$ $30-20-10-30 \mathrm{~km}$ under different node failure scenarios are summarized in Table VII. The results confirm that the amplifier placement solution for the self-healing ring in Fig. 8 still works for a single node failure.

\section{CONCLUSION}

In this paper, two novel amplifier placement methods for metropolitan WDM ring networks were presented. Method 1 uses an MINLP problem to exactly describe the network and device requirements and solves the problem. The solution includes the number of amplifiers in the rings, the gain and location of each amplifier on each link, together with the transmitted powers of channels at each node. Although the solution is not guaranteed to be optimal due to the highly nonlinear nature of some constraints, the solution is efficient and provides an effective guideline for the ring network design. It was found 
TABLE VII

Minimum Amplifier Gains for a SiX-Node Self-HeAling Ring With Link Lengths $1-2-3-4-5-6=10-20-30-20-10-30 \mathrm{~km}$

UNDER DIFFERENT NODE FAILURES

\begin{tabular}{c|c|c|c|c|c|c|c}
\hline $\begin{array}{c}\text { Amp. } \\
\text { No. }\end{array}$ & \multicolumn{7}{|c}{ Amplifier Gain (dB) } \\
\cline { 2 - 8 } & $\begin{array}{c}\text { No } \\
\text { failure }\end{array}$ & $\begin{array}{c}\text { Node 1 } \\
\text { fails }\end{array}$ & $\begin{array}{c}\text { Node 2 } \\
\text { fails }\end{array}$ & $\begin{array}{c}\text { Node 3 } \\
\text { fails }\end{array}$ & $\begin{array}{c}\text { Node 4 } \\
\text { fails }\end{array}$ & $\begin{array}{c}\text { Node 5 } \\
\text { fails }\end{array}$ & $\begin{array}{c}\text { Node 6 } \\
\text { fails }\end{array}$ \\
\hline $\mathbf{A}_{\mathbf{1}}$ & 7.4 & 7.6 & Unused & 5.5 & 5.1 & 11.7 & 7.7 \\
\hline $\mathbf{A}_{\mathbf{2}}$ & 13.4 & 9.2 & 0 & Unused & 13.1 & 12.2 & 10.9 \\
\hline $\mathbf{A}_{\mathbf{3}}$ & 12.1 & 12.1 & 15 & 0 & Unused & 14 & 10.4 \\
\hline $\mathbf{A}_{\mathbf{4}}$ & 11 & 7.7 & 4.8 & 7.1 & 0 & Unused & 8.8 \\
\hline $\mathbf{A}_{\mathbf{5}}$ & 9.7 & 9.2 & 10.9 & 9.3 & 8 & 2.9 & Unused \\
\hline $\mathbf{A}_{\mathbf{6}}$ & 14.4 & Unused & 10.4 & 11.1 & 13.4 & 7.9 & 0 \\
\hline $\mathbf{A}_{\mathbf{8}}$ & Unused & 18.6 & 10.1 & 7 & 0 & Unused & 16.9 \\
\hline $\mathbf{A}_{\mathbf{1 2}}$ & Unused & Unused & 16.8 & 19 & 21.4 & 26.1 & 9.2 \\
\hline
\end{tabular}

that by placing the amplifier at the end of the link, the required number of amplifiers in the network was not significantly higher than that required by placing the amplifier anywhere on the link. In addition, placing the amplifier at the end of the link facilitates network maintenance and reduces cost. The amplifier placement Method 2 using the MILP programming technique was also presented. The solution is guaranteed to be optimal. However, due to the approximation process, the linear constraints are not precise and are stricter than the original nonlinear constraints. Consequently, the solution from the MILP problem has more amplifiers than that from the MINLP problem. Both amplifier placement Methods 1 and 2 can be used in conjunction to solve the amplifier placement problem in a ring network.

A new amplifier placement method (the iterative method) for self-healing WDM rings was also presented. The method is based on iteratively solving an amplifier placement problem for a ring network under a normal and different link-failure scenarios. The amplifier placement solution derived using the iterative method ensures the self-healing ring network is operational under a normal condition or any single link or node failures.

With the optimization of the number of amplifiers using the amplifier placement methods presented in this paper, the number of amplifiers in the ring network is greatly reduced compared with the common method of placing one amplifier at every node. The methods enable the design of metropolitan WDM ring networks with low cost while maintaining a good performance.

\section{REFERENCES}

[1] A. A. M. Saleh and J. M. Simmons, "Architectural principles of optical regional and metropolitan access networks," J. Lightwave Technol., vol. 17, pp. 2431-2448, Dec. 1999.

[2] M. Kuznetsov, N. M. Froberg, S. R. Henion, H. G. Rao, J. Korn, K. A. Rauschenbach, E. H. Modiano, and V. W. C. Chan, "A next-generation optical regional access network," IEEE Commun. Mag., vol. 38, pp. 66-72, Jan. 2000

[3] S. S. Wagner and T. E. Chapuran, "Multiwavelength ring networks for switch consolidation and interconnection," in Proc. Int. Communications Conf. (ICC 1992), 1992, pp. 1173-1179.

[4] A. F. Elrefaie, "Multiwavelength survivable ring network architectures," in Proc. Int. Communications Conf. (ICC 1993), 1993, pp. 1245-1251.

[5] C.-S. Li, F. F.-K. Tong, C. J. Georgiou, and M. Chen, "Gain equalization in metropolitan and wide area optical networks using optical amplifiers," presented at the IEEE INFOCOM'94, Los Alamitos, CA, 1994, pp. $1 \mathrm{~d} .3 .1-1 \mathrm{~d} .3 .8$
[6] B. Ramamurthy, J. Iness, and B. Mukherjee, "Optimizing amplifie placements in a multiwavelength optical LAN/MAN: The equally powered-wavelength case," J. Lightwave Technol., vol. 16, pp. 1560-1569, Sept. 1998.

[7] - "Optimizing amplifier placements in a multiwavelength optical LAN/MAN: The unequally powered-wavelength case," IEEE/ACM Trans. Networking, vol. 6, pp. 755-767, June 1998.

[8] J. Iness and B. Mukherjee, "New optical amplifier placement schemes for broadcast networks," Eur. Trans. Telecommunications \& Related Technologies, vol. 11, pp. 117-124, Jan.-Feb. 2000.

[9] A. Fumagalli, G. Balestra, and L. Valcarenghi, "Optimal amplifier placement in multi-wavelength optical networks based on stimulated annealing," in Proc. Conf. All-Optical Networking: Architecture, Control, Management Issues, Boston, MA, 1998, pp. 268-279.

[10] L. Zhong and B. Ramamurthy, "Optimization of amplifier placements in switch-based optical networks," in Proc. Int. Conf. Communications (ICC 2001), 2001, pp. 224-228.

[11] C. R. Giles and M. Spector, "The wavelength add/drop multiplexer for lightwave communication networks," Bell Labs Tech. J., pp. 207-229, Jan.-Mar. 1999.

[12] M. Peroni and M. Tamburrini, "Gain in erbium-doped fiber amplifiers: A simple analytical solution for the rate equations," Opt. Lett., vol. 15, no. 15 , pp. 842-844, Aug. 1990.

[13] R. Ramaswami and K. Liu, "Analysis of effective power budget in optical bus and star networks using erbium-doped fibers amplifiers," $J$. Lightwave Technol., vol. 11, pp. 1863-1871, Nov. 1993.

[14] W. L. Winston, Operations Research: Applications and Algorithms: Duxbury Press, 1994, sec. 9.2.

[15] W. D. Cornwell and I. Andonovic, "Interferometric noise for a single interferer: comparison between theory and experiment," Electron. Lett., vol. 32, pp. 1501-1502, 1996.

[16] G. L. Nemhauser and L. A. Wolsey, Integer and Combinatorial Optimization. New York: Wiley, 1988, sec. I.5.

[17] C. A. Floudas, Nonlinear and Mixed-Integer Optimization. New York: Oxford Univ. Press, 1995, ch. 6.

[18] Nonlinear Programming Frequently Asked Questions. [Online]http://www-unix.mcs/anl.gov/otc/Guide/faq/nonlinear-programming-faq.html

[19] R. Fourer, D. M. Gay, and B. W. Kernighan, AMPL: A Modeling Language for Mathematical Programming. San Francisco, CA: Scientific, 1993.

[20] MINLP Solver Running on NEOS Server. [Online]http://wwwneos.mcs.anl.gov/neos/solvers/IP:MINLP-AMPL/

[21] R. Fletcher and S. Leyffer, "Numerical experience with lower bounds for MIQP branch-and-bound," SIAM J. Optim., vol. 8, no. 2, pp. 604-616, 1998.

[22] —, User Manual for FilterSQP [Online]. Available: http://www.maths.dundee.ac.uk/

[23] E. M. L. Beale, "Integer programming," in The State of the Art in Numerical Analysis, D. A. H. Jacobs, Ed. New York: Academic, 1978.

[24] S. Leyffer. User Manual for MINLP_BB [Online]. Available: http://www.maths.dundee.ac.uk/

[25] G. L. Nemhauser, A. H. G. R. Kan, and M. J. Todd, Optimization: Handbooks in Operations Research and Management Science. New York: North-Holland, 1989. 
[26] A. L. Peressini, F. E. Sullivan, and J. J. Uhl Jr., The Mathematics of Nonlinear Programming. New York: Springer-Verlag, 1988.

[27] XPRESS Solver Running on NEOS Server. [Online]. Available: http://www-neos.mcs.anl.gov/neos/solvers/MILP:XPRESS-AMPL/

[28] Dash Associates. XPRESS-Modeling and Optimization Software. [Online]. Available: http://www.dash.co.uk

[29] T.-H. Wu and R. C. Lau, "A class of self-healing ring architectures for SONET network applications," IEEE Trans. Commun., vol. 40, pp. 1746-1756, Nov. 1992.

[30] T.-H. Wu and W. I. Way, "A novel passive protected SONET bidirectional self-healing ring architecture," J. Lightwave Technol., vol. 10, pp. 1314-1322, Sept. 1992.

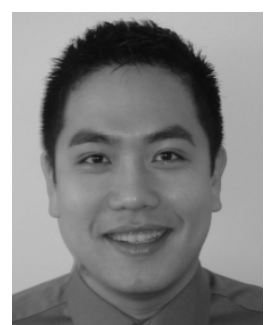

An V. Tran (S'99-M'04) received the B.E. degree in communications engineering (First-Class Hons.) from the Royal Melbourne Institute of Technology (RMIT), Melbourne, Australia, in 1998 and the Ph.D. degree in electrical and electronic engineering from the University of Melbourne, Melbourne, Australia, in 2002, with a dissertation on novel optical add-drop multiplexers and optimization of metropolitan wavelength-division-multiplexing (WDM) ring networks.

From 2002 to 2003, he was with the Photonics Research Laboratory, University of Melbourne, working on new subsystems for bidirectional optical networks. In 2004, he joined the ARC Special Research Centre for Ultra-Broadband Information Networks (CUBIN) at the University of Melbourne, where he worked on optical packet switching and optical networking. Since 2003, he has also been a Project Manager for an industry contract between Telstra Corporation and the University of Melbourne, researching various aspects of long-haul dense-wavelength-division-multiplexing (DWDM) systems and automatically switched optical networks (ASONs). His research interests include designs of new subsystems and architectures for optical packet/burst switching, optical access networks, and the internetworking study of Internet protocol over optical transport networks.

Dr. Tran received the Ericsson Prize in 1995 and the MM Cables Prize in 1997, both from RMIT. From 1998 to 2002, he was awarded the IPRS scholarship from the University of Melbourne.

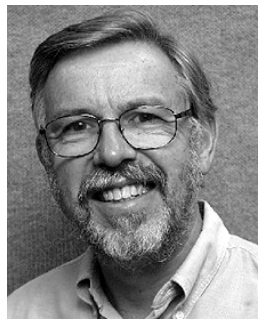

Rodney S. Tucker (S'72-M'75-SM'85-F'90) received the B.E. degree in electronics and the Ph.D. degree from the University of Melbourne, Melbourne, Australia, in 1969 and 1975, respectively.

He was a Lecturer in Electrical Engineering at the University of Melbourne from 1973 to 1975 . From 1975 and 1976, he was a Harkness Fellow with the Department of Electrical Engineering and Computer Sciences, University of California, Berkeley, and from 1976 to 1977 he was a Harkness Fellow with the School of Electrical Engineering, Cornell University, Ithaca, New York. From 1977 to 1978 he was with Plessey Research (Caswell) Ltd., U.K., and from 1978 to 1983, he was with the Department of Electrical Engineering at the University of Queensland, Brisbane, Australia. From 1984 to 1990, he was with AT\&T Bell Laboratories, Crawford Hill Laboratory, Holmdel, NJ, and in 1990 he joined the Department of Electrical and Electronic Engineering at the University of Melbourne, where he is the Founding Director of the ARC Special Research Centre for Ultra-Broadband Information Networks. He is also associated with the Australian Photonics Cooperative Research Centre.

Prof. Tucker is a Fellow of the Australian Academy of Science and a Fellow of the Australian Academy of Technological Sciences and Engineering. He served on the Management Committee of the Australian Telecommunications and Electronics Research Board from 1991 to 1993 and has been a member of the Australasian Council on Quantum Electronics. From 1995 to 1999, he served as a Member of the Board of Governors of the IEEE Lasers and Electro-Optics Society (LEOS). He was awarded the Fisk Prize by the Institution of Radio and Electronics Engineers, Australia, in 1970, and in 1975 he received a Harkness Fellowship by the Commonwealth Fund, New York. He has been awarded the Institution of Engineers, Australia, M.A. Sargent Medal for 1995 and was named an IEEE Lasers and Electro-Optics Society Distinguished Lecturer for the year 1995-1996. He also received the Australia Prize for his contributions to telecommunications in 1997, an ARC Federation Fellowship in 2002, and a Centenary Medal in 2003. In 2004, the international citation register ISI recognized him as one of the 200 most cited authors in international engineering literature.

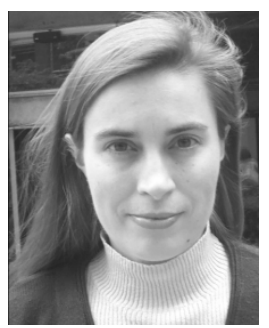

Natashia L. Boland received the B.Sc. (Hons.) degree in mathematics and computer science and the $\mathrm{Ph} . \mathrm{D}$. degree in mathematics from the University of Western Australia in 1988 and 1992, respectively.

She worked with a Melbourne software company, The Preston Group, on airline applications of operations research for part of 1992. She then held a postdoctoral research fellowship at the School of Industrial and Systems Engineering, Georgia Institute of Technology, Atlanta, in 1994 and a postdoctoral research fellowship at the Department of Combinatorics and Optimization at the University of Waterloo, Ottawa, ON, Canada, in 1993. She is currently a Senior Lecturer in the Department of Mathematics and Statistics at the University of Melbourne, Melbourne, Australia, and a member of the Department's Operations Research Group. She has regularly provided consulting services to the industry in a number of areas, particularly in airline planning, transportation logistics, and scheduling. With the creation of Melbourne Operations Research-MORe, which aims to deliver consulting services and expertise in operations research to business, industry, government and community organizations, she is now dedicated, as its director, to the delivery of professional consulting services. 